Revista Multidisciplinar do Nordeste Mineiro, v.2 2019/02

ISSN 2178-6925

\title{
CRESCIMENTO E NUTRIÇÃO MINERAL DE PLANTAS DE ARROZ EXPOSTAS AO CROMO
}

\author{
GROWTH AND MINERAL NUTRITION OF RICE PLANTS EXPOSED TO \\ CHROMIUM
}

\begin{abstract}
Viviane Amaral Toledo Coelho
Doutora. Autor parar correspondência; vivianeatc@yahoo.com.br. Rua Rodrigues Seabra, 243, Centro - Almenara - MG CEP: 39900-000. Alfa - Faculdade de Almenara, Almenara/MG, Brasil.

Carla Giselly de Souza

Doutora. Universidade Federal Rural de Pernambuco - UFRPE, Recife, Pernambuco, Brasil.

Luiza Gobira Lacerda

Especialista. Alfa - Faculdade de Almenara, Almenara, Minas Gerais, Brasil.

Mônica Cecília Santana Pereira

Doutora. Alfa - Faculdade de Almenara, Almenara, Minas Gerais, Brasil.
\end{abstract}

\section{Resumo}

Objetivo: avaliar o teor e o acúmulo de macro e micronutrientes e de $\mathrm{Cr}$ em plantas de arroz expostas ao cromo em solução nutritiva. O experimento foi realizado em casa de vegetação do DCS/UFLA, Lavras-MG. Material e Métodos: o delineamento estatístico utilizado foi o DIC com quatro repetições, contendo seis tratamentos, em solução nutritiva. Os tratamentos foram: $0 ; 0,04 ; 0,08 ; 0,16 ; 0,32$ e $0,64 \mathrm{mmol} \mathrm{L}^{-1} \mathrm{de}$ $\mathrm{Cr}\left[\mathrm{Cr}\left(\mathrm{NO}_{3}\right)_{3} \cdot 9 \mathrm{H}_{2} \mathrm{O}\right]$. Após a germinação, as mudas foram transferidas para a solução nutritiva completa com $10 \%$ da sua força iônica (período de adaptação), as quais permaneceram sob aeração constante. Após a adaptação, as plantas foram selecionadas quanto à uniformidade de tamanho da parte aérea e raízes e transplantadas para vasos de plástico (três litros) com solução nutritiva a $100 \%$, nos quais foram estabelecidos os tratamentos. Por ocasião da colheita das plantas, as mesmas foram divididas em parte aérea, inflorescência e raiz, sendo as partes lavadas e secas em estufa; após a secagem realizou-se a obtenção da massa de matéria seca. Após a pesagem, procedeu-se à moagem do material para posterior análise química. Resultados: Após 15 dias em solução nutritiva, as plantas com as doses acima de $0,08 \mathrm{mmol} \mathrm{L}^{-1}\left(4,16 \mathrm{mg} \mathrm{L}^{-1}\right)$ começaram a apresentar sintomas de toxidez, havendo redução do crescimento do sistema radicular e parte aérea. Os teores e acúmulos $\mathrm{s}$ de $\mathrm{Cr}$ e micronutrientes na raiz, parte aérea e inflorescências foram influenciados de forma significativa pela presença de $\mathrm{Cr}$ em solução nutritiva. Conclusões: $\mathrm{O}$ aumento da concentração de $\mathrm{Cr}$ em solução nutritiva provoca diminuição na produção de massa de matéria seca de arroz. Os teores e acúmulos de nutrientes e $\mathrm{Cr}$ na raiz, parte aérea e inflorescência são influenciados pela presença de $\mathrm{Cr}$ em solução nutritiva, mostrando respostas diferenciadas.

Palavras-chave: elementos-traço; Oryza sativa; contaminação.

\section{Abstract}

Objective: To evaluate the content and accumulation of macro and micronutrients and $\mathrm{Cr}$ in rice plants exposed to chromium in nutrient solution. The experiment was carried out in a greenhouse of DCS / UFLA, Lavras-MG. Material and Methods: The 
statistical design used was the four-repetition totally random, containing six treatments, in a nutrient solution. The treatments were: $0 ; 0.04 ; 0.08 ; 0.16 ; 0.32$ and $0.64 \mathrm{mmol} \mathrm{L}{ }^{-1}$ of $\mathrm{Cr}\left[\mathrm{Cr}\left(\mathrm{NO}_{3}\right)_{3} \cdot 9 \mathrm{H}_{2} \mathrm{O}\right]$. After germination, the seedlings were transferred to the complete nutrient solution with $10 \%$ of their ionic strength (adaptation period), which remained under constant aeration. After adaptation, the plants were selected for uniformity of shoot and root size and transplanted to plastic pots (three liters) with $100 \%$ nutrient solution, in which treatments were established. At the time of harvesting the plants were divided into shoots, inflorescences and roots, and the parts were washed and dried in the greenhouse; After drying, the dry matter mass was obtained. After weighing, the material was milled for later chemical analysis. Results: After 15 days in nutrient solution, plants with doses above 0.08 $\mathrm{mmol} \mathrm{L}^{-1}\left(4.16 \mathrm{mg} \mathrm{L}^{-1}\right)$ began to show symptoms of toxicity, with reduction of root and shoot growth. $\mathrm{Cr}$ and micronutrient contents and accumulations in root, shoot and inflorescences were significantly influenced by the presence of $\mathrm{Cr}$ in nutrient solution. Conclusions: Increasing $\mathrm{Cr}$ concentration in nutrient solution causes decrease in rice dry matter mass production. Nutrient and accumulation contents and $\mathrm{Cr}$ in root, shoot and inflorescence are influenced by the presence of $\mathrm{Cr}$ in nutrient solution, showing different responses.

Key-words: trace elements; Oryza sativa; contamination.

\section{Introdução}

No Brasil, o cultivo arroz (Oriza sativa L.) é difundido nacionalmente, e possui grande importância econômica e social, por ser um produto básico da alimentação da população (junto com o feijão) e ser cultivado em todas as Unidades da Federação (FERREIRA et al., 2009). Tratar do arroz no Brasil é mais do que abordar atividades desenvolvidas nas dimensões econômicas, agronômicas e comerciais ao longo da cadeia produtiva (FERREIRA; VILAR, 2018).

Atualmente o cultivo do arroz, é realizado com alta tecnologia, exigindo, portanto, um intenso uso de insumos. A utilização excessiva de fertilizantes e herbicidas associados ao grande volume de água vem promovendo impactos ambientais negativos (FERREIRA; VILAR, 2018).

Muitos estudos são conduzidos para diagnosticar o comportamento de distintas espécies submetidas à contaminação por metais pesados. A poluição do solo e de sistemas aquáticos por elementos-traço (também chamados de metais pesados) é um fator que afeta a qualidade do meio ambiente e constitui risco eminente de intoxicação ao homem. Nos últimos anos, foram realizadas inúmeras pesquisas com a finalidade de avaliar os possíveis impactos ambientais relacionados ao aumento da concentração de elementos-traço no meio ambiente. Esses elementos são originários de processos litogênicos e/ou atividades 
antrópicas, como a utilização de fertilizantes em zonas agrícolas e a atividade mineradora (MUNIZ; OLIVEIRA-FILHO, 2006).

O Cr é encontrado naturalmente em solos, águas, rochas, cinzas vulcânicas e gases e utilizado em diversas aplicações industriais como na galvanização, na preservação da madeira, na fabricação de corantes, tintas e papel, no refino do petróleo, no curtimento de couros, entre outras aplicações (GUPTA; RASTOGI; NAYAK, 2010; KABATA-PENDIAS; MUKHERJEE, 2007).

O Cromo é um elemento químico encontrado em múltiplas valências, com diferenças marcantes entre elas. Na natureza, o $\mathrm{Cr}$ ocorre tanto na forma trivalente $\left(\mathrm{Cr}^{3+}\right)$ como na forma hexavalente $\left(\mathrm{Cr}^{6+}\right)$. Devido à sua maior solubilidade, o $\mathrm{Cr}^{6+}$ mostra maior toxicidade do que o $\mathrm{Cr}^{3+}$. $\mathrm{O} \mathrm{Cr}^{3+}$ tem sido considerado um elemento estável no solo (LOPES, 2013).

A partir de 1959, o Cr passou a ser considerado um elemento essencial aos seres humanos. A deficiência de cromo em humanos e outros mamíferos tem sintomas comparados àqueles associados ao início de diabetes e doenças cardiovasculares, como; elevação da glicose sanguínea, insulina, colesterol e triglicerídeos e diminuição das lipoproteínas de alta densidade (HDL). O consumo do cromo (picolinato de cromo) aumenta a atividade da insulina e tem sido objeto de estudos que avaliam o metabolismo de carboidratos, proteínas e lipídeos. Os efeitos sugeridos incluem aumento da massa magra, diminuição do percentual de gordura e aumento da taxa de metabolismo basal (MARANGON; FERNANDES, 2005).

Embora o $\mathrm{Cr}$ não possua a sua essencialidade comprovada na nutrição vegetal, é um componente essencial da nutrição humana e animal. Estudos indicam que pequenas concentrações do metal (cerca de $1 \mu \mathrm{M}$ ) podem estimular 0 crescimento dos vegetais, sendo tóxico para a maioria das culturas em níveis entre 0,5 a $5,0 \mu \mathrm{g} \mathrm{mL}^{-1}$ em solução nutritiva, e de 5 a $100 \mu \mathrm{g} \mathrm{g}^{-1}$ em solos (CIPRIANI, 2008).

A maioria das espécies vegetais crescendo em solos contaminados por elementos-traço não consegue evitar a absorção desses elementos, mas somente limitar sua translocação. Ainda que existam muitas incertezas sobre a especificidade dos mecanismos de absorção desses elementos, sobretudo daqueles não essenciais, geralmente o teor e o acúmulo do elemento nos tecidos são em função de sua disponibilidade na solução do solo e os teores nas raízes e parte aérea aumentam com a elevação da concentração de metais na solução do solo (SOARES 
et al., 2001). Diante do exposto, o objetivo desse trabalho foi avaliar o crescimento e nutrição mineral de plantas de arroz expostas ao cromo, em solução nutritiva.

\section{MATERIAL E MÉTODOS}

O ensaio foi conduzido em casa de vegetação, no Departamento de Ciência do Solo, localizado na Universidade Federal de Lavras, Lavras - MG. As sementes de arroz (cultivar Conai) foram germinadas em substrato (vermiculita) e após 15 dias, as plantas foram transferidas para bandeja com capacidade de 36 litros, contendo solução nutritiva de Hoagland e Arnon (1950) a 75\% de sua força iônica para a fase de adaptação, na qual permaneceram também por 15 dias.

As plântulas foram selecionadas quanto à uniformidade de tamanho da parte aérea e raízes. Posteriormente, foram transplantadas para vasos de plástico (3 litros) com solução nutritiva (HOAGLAND; ARNON, 1950) a 100\%. Os tratamentos foram distribuídos em delineamento experimental inteiramente casualizado (DIC), com quatro repetições. A troca de solução nutritiva com diferentes doses de cromo foi realizada quinzenalmente, e nos intervalos, o volume dos vasos foi completado com água deionizada, sempre que necessário. As doses de $\mathrm{Cr}\left[\mathrm{Cr}\left(\mathrm{NO}_{3}\right)_{3} \cdot 9 \mathrm{H}_{2} \mathrm{O}\right]$ aplicadas foram: $0 ; 0,04 ; 0,08 ; 0,16 ; 0,32$ e 0,64 $\mathrm{mmol} \mathrm{L}^{-1}$.

Após 60 dias (início do florescimento), as plantas foram colhidas e lavadas em água destilada. A parte aérea, inflorescências e raízes foram colocadas, separadamente, em sacos de papel e submetidas à secagem em estufa a $65^{\circ} \mathrm{C}$, durante 72 horas, para a determinação da matéria seca.

Após a secagem, procedeu-se também à moagem do material para posterior análise química, determinando-se os teores nutricionais e de $\mathrm{Cr}$, de acordo com os métodos descritos por Malavolta, Vitti e Oliveira (1997).

Através do índice de translocação (IT) é possível avaliar a capacidade das espécies em translocar o Cr da raiz para a parte aérea, conforme Paiva et al.(2002), que é calculado pela expressão:

$$
I T=\frac{\text { conteúdodo elemento na parte aérea }}{\text { conteúdodo elemento na planta }} \times 100
$$

Os dados foram submetidos à análise da variância e regressão polinomial, a $5 \%$ de probabilidade. Os critérios para escolha dos modelos de regressão foram aqueles que mostraram maior coeficiente de determinação, significância dos coeficientes de regressão até $5 \%$ de probabilidade, pelo teste $t$ e significado 
biológico do modelo. As análises estatísticas foram realizadas com o auxílio do programa computacional Sisvar (FERREIRA, 2010).

\section{RESULTADOS E DISCUSSÃO}

Após 15 dias em solução nutritiva, as plantas com as doses acima de 0,08 mmol L-1 (4,16 $\left.\mathrm{mg} \mathrm{L}^{-1}\right)$ começaram a apresentar sintomas de toxidez, havendo redução do crescimento do sistema radicular e parte aérea. Cipriani (2008), estudando doses crescentes do $\operatorname{Cr}(0,00$ (controle); 0,$04 ; 0,08 ; 0,16 ; 0,32$; e 0,64 $\mathrm{mM}$ ) no desenvolvimento de mudas de eucalipto (Eucalyptus urograndis). Verificaram que a matéria seca de raízes sofreu redução de $38,76 \% ; 48,22 \%$; 64,50\%; e 70,41\% em relação ao controle nas concentrações crescentes de cromo, respectivamente.

Ferreira (2007) revela que, os sintomas da toxicidade do $\mathrm{Cr}$ em plantas são evidenciados por uma redução do crescimento, desenvolvimento rudimentar do sistema radicular, murchidão foliar, descoloração, clorose e crescimento irregular das folhas. Shanker et al. (2005), complementa que tal efeito se deve possivelmente a inibição de divisão e elongamento das células radiculares ou a extensão do ciclo celular nas raízes.

Para o peso da matéria seca da raiz, da parte aérea e total houve resposta linear negativa com aumento da concentração de $\operatorname{Cr}$ (Figuras 1, 2 e 3).

Figura 1. Massa de matéria seca da parte aérea (g) de plantas de arroz em diferentes doses de cromo, Lavras-MG.

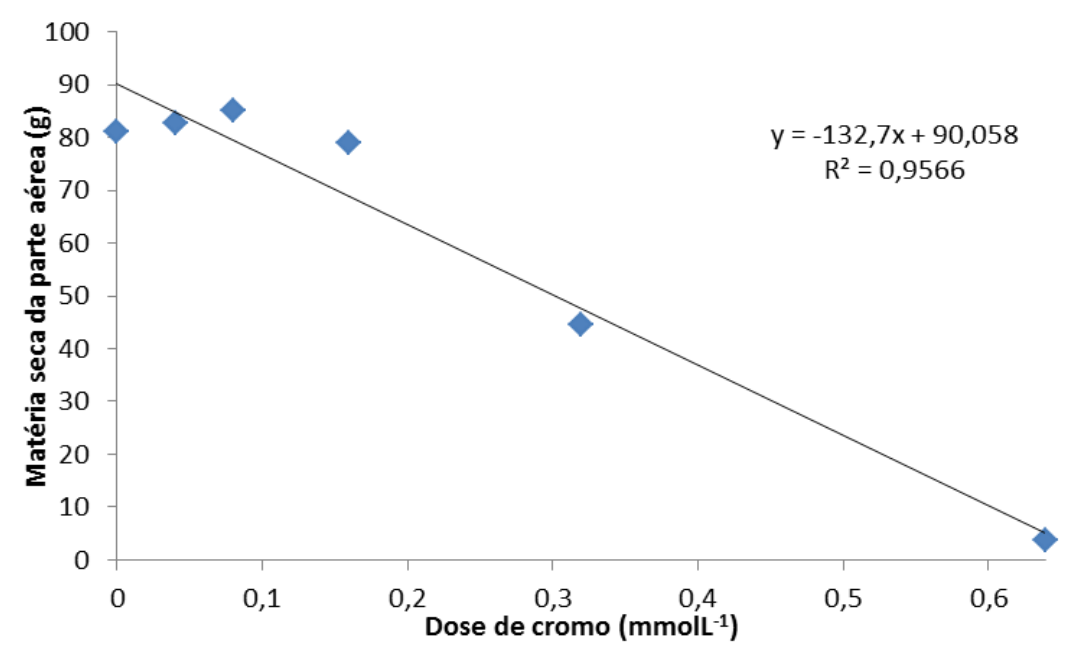

Fonte: próprios autores. 
Figura 2. Massa de matéria seca da raiz (g) de plantas de arroz em diferentes doses de cromo, Lavras-MG.

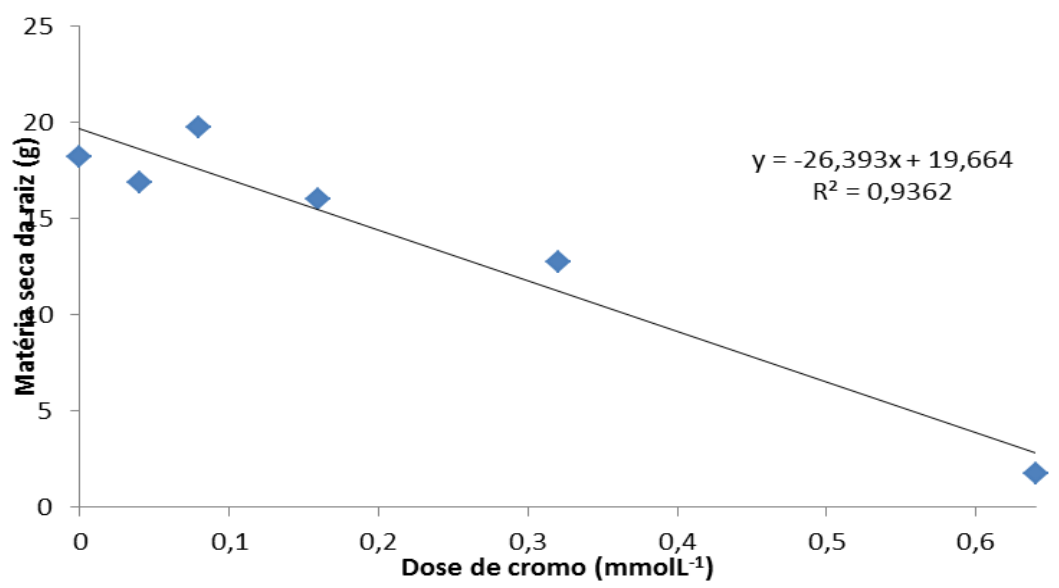

Fonte: próprios autores.

Figura 3. Massa de matéria seca total ( $\mathrm{g}$ ) de plantas de arroz em diferentes doses de cromo, Lavras-MG.

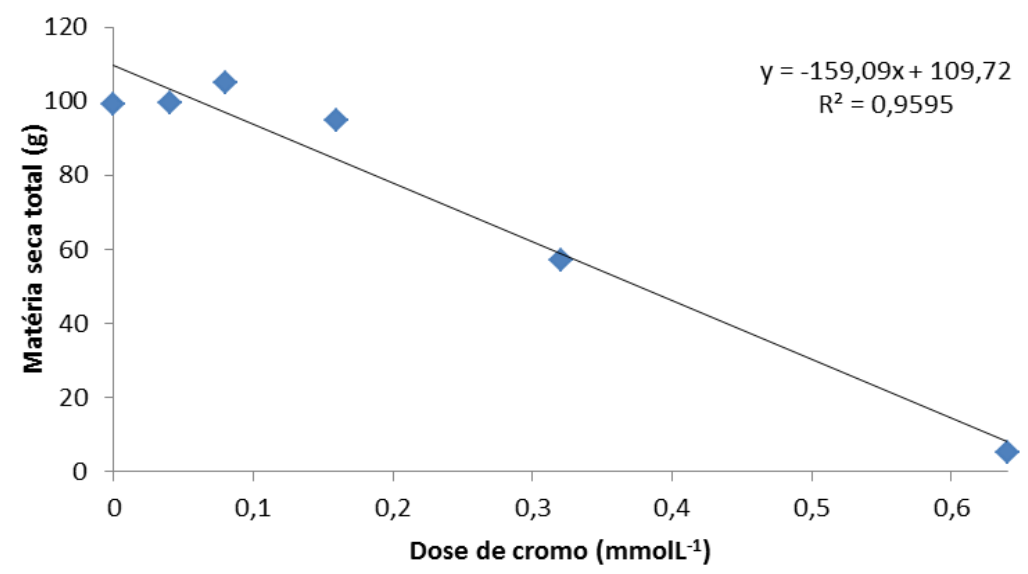

Fonte: próprios autores.

Sintomas similares foram observados em plantas de soja submetidas a doses superiores a $13 \mathrm{mg} \mathrm{L}^{-1} \mathrm{Cr}^{6+}$ (CASTILHOS; GUADAGNIN; SILVA, 2001ab). Ainda, para esses autores, as massas de matéria seca foliar e radicular não foram afetadas pela presença de $5 \mathrm{mg} \mathrm{L}^{-1} \mathrm{Cr}^{6+}$, quando comparados ao tratamento controle.

Castilhos; Guadagnin; Silva (2001b) observaram, ainda, que a massa de matéria seca de folhas e raízes de soja não foram afetadas pela presença de $20 \mathrm{mg}$ 
$\mathrm{L}^{-1} \mathrm{Cr}^{3+}$ cujos valores foram semelhantes ao controle. Acima dessa concentração a massa de matéria seca das plantas foi afetada com o aumento da dose de $\mathrm{Cr}^{3+}$.

Ferreira (2007) trabalhando com três variedades de alface Lactuca sativa $\mathrm{L}$. Twistil (Frisada), Teide (Roxa), Povoa (Lisa), expostos a concentrações de 0 (controle), 20, 50, 100, 150, 200 e $300 \mathrm{mg} \mathrm{Cr} / \mathrm{L}$ por 30 dias. Onde observaram que a partir de 10 dias de exposição a cromo, as plantas começaram a apresentar sintomas de toxicidade (e.g. clorose e necrose) que se prolongaram (e em geral, agravaram) ao longo do ensaio. Onde a variedade Twistil apresentava sinais claros de nanismo nas concentrações mais elevadas concentrações $\left(150,200 \mathrm{mg} \mathrm{L}^{-1}\right.$ ) assim como manchas necróticas e emurchecimento.

Bonfranceschi, Flocco e Dinati (2009) estudando a aplicação de $\mathrm{Cr}^{3+}$ e $\mathrm{Cr}^{6+}$ em plantas de sorgo e alfafa, verificaram que ambas as espécies tiveram menor produção de matéria seca nas maiores doses de $\mathrm{Cr}\left(80 \mathrm{mg} \mathrm{L}^{-1}\right.$ para $\mathrm{Cr}^{3+}$ e $10 \mathrm{mg} \mathrm{L}^{-1}$ para $\left.\mathrm{Cr}^{6+}\right)$.

Em altas concentrações, o $\mathrm{Cr}$ pode causar estresse oxidativo, induzindo a degradação de pigmentos fotossintéticos, levando à diminuição do crescimento. $E$ ainda pode causar distúrbios à estrutura dos cloroplastos e, consequentemente, afetar o processo fotossintético (PANDA; CHOUDHURY, 2005).

Os teores de macronutrientes na raiz, parte aérea e inflorescências foram influenciados de forma significativa pela presença de $\mathrm{Cr}$ em solução nutritiva e são mostrados na Tabela 1.

Tabela 1. Equações de regressão para os teores de macronutrientes na raiz, parte aérea e inflorescência de plantas de arroz, em resposta a doses de cromo ( 0 a 64 mmol L-1 Cr). UFLA, Lavras-MG. 


\begin{tabular}{|c|c|c|c|}
\hline Nutriente & Parte da planta & Equação de regressão & $\mathrm{R}^{2}$ \\
\hline \multirow[t]{3}{*}{$\mathrm{N}$} & Raiz & $\begin{array}{l}y=17,18075- \\
14,837130 x+123,315692 x^{2}\end{array}$ & 0,99 \\
\hline & Parte aérea & $\begin{array}{l}y=21,598047+49,601573 x- \\
66,998225 x^{2}\end{array}$ & 0,87 \\
\hline & Inflorescência & $\begin{array}{l}y=20,288206+43,732264 x- \\
116,99455 x^{2}\end{array}$ & 0,96 \\
\hline \multirow{3}{*}{$P$} & Raiz & $y=2,802078+19,286179 x-25,535797 x^{2}$ & 0,89 \\
\hline & Parte aérea & s.a. & - \\
\hline & Inflorescência & $y=3,132883-4,012736 x+12,043615 x^{2}$ & 0,97 \\
\hline \multirow{3}{*}{$\mathrm{K}$} & Raiz & s.a. & \\
\hline & Parte aérea & $y=20,202857-8,562212 x$ & 0,70 \\
\hline & Inflorescência & $y=6,439687-4,161920 x+20,019531 x^{2}$ & 0,99 \\
\hline \multirow{3}{*}{$\mathrm{Ca}$} & Raiz & $y=4,139062-0,464592 X-8,716605 X^{2}$ & 0,89 \\
\hline & Parte aérea & $y=4,939643-3,008353 x$ & 0,77 \\
\hline & Inflorescência & $y=0,918023-2,469912 X+3,715269 X^{2}$ & 0,86 \\
\hline \multirow{3}{*}{$\mathrm{Mg}$} & Raiz & $y=1,683875+0,076764 X-2,828881 X^{2}$ & 0,96 \\
\hline & Parte aérea & $y=4,601357-3,597293 x$ & 0,91 \\
\hline & Inflorescência & $y=1,732859+1,701061 x-3,508012 x^{2}$ & 0,87 \\
\hline \multirow{3}{*}{ S } & Raiz & s.a. & - \\
\hline & Parte aérea & $y=2,094523+5,249644 x-13,245416 x^{2}$ & 0,98 \\
\hline & Inflorescência & $y=1,474078+2,387389 x-7,239426 x^{2}$ & 0,91 \\
\hline
\end{tabular}

** significativo a $5 \%$ de probabilidade; n.s. não-significativo a $5 \%$ de probabilidade; e s.a. sem ajuste. Fonte: próprios autores.

$\mathrm{O}$ teor radicular de $\mathrm{N}$ apresentou resposta quadrática à aplicação de $\mathrm{Cr}$, tendo alcançado o máximo na dose de 0,06 $\mathrm{mmol} \mathrm{L}^{-1}$. Os teores radiculares de $\mathrm{P}, \mathrm{Ca}$ e $\mathrm{Mg}$ também apresentaram resposta quadrática, com os pontos mínimos nas doses de 0,38; 0,14 e $0,01 \mathrm{mmol} \mathrm{L}^{-1}$ de $\mathrm{Cr}$.

Os teores de $\mathrm{N}$ e $\mathrm{S}$ da parte aérea das plantas de arroz alcançaram o mínimo nas doses de 0,37 e 0,20 $\mathrm{mmol} \mathrm{L}^{-1}$ de $\mathrm{Cr}$. Já os teores da parte aérea de $\mathrm{K}, \mathrm{Ca}$ e $\mathrm{Mg}$ diminuíram com o aumento de $\mathrm{Cr}$ em solução nutritiva.

Para os teores de macronutrientes da inflorescência, todos apresentaram resposta quadrática. Os teores de $\mathrm{N}, \mathrm{Mg}$ e $\mathrm{S}$ obtiveram os mínimos nas doses de 0,19; 0,24 e 0,16 mmol L-1 de Cr. Para os teores de P, K, Ca e Mg, os mínimos 
foram obtidos nas doses 0,$17 ; 0,10$ e $0,13 \mathrm{mmol} \mathrm{L}^{-1}$ de $\mathrm{Cr}$. Os teores de $\mathrm{Cr}$ e micronutrientes na raiz, parte aérea e inflorescências foram influenciados de forma significativa pela presença de $\mathrm{Cr}$ em solução nutritiva e são mostrados na Tabela 2.

Tabela 2. Equações de regressão para os teores de micronutrientes e de cromo na raiz, na parte aérea e inflorescência de plantas de arroz, em resposta a doses de cromo (0 a $64 \mathrm{mmol} \mathrm{L}^{-1} \mathrm{Cr}$ ). UFLA, Lavras-MG.

\begin{tabular}{|c|c|c|c|}
\hline Nutriente & Parte da planta & Equação de regressão & $\mathrm{R}^{2}$ \\
\hline \multirow{3}{*}{ B } & Raiz & $y=15,657312+46,973480 x-111,887811 x^{2}$ & 0,98 \\
\hline & Parte aérea & $y=26,040286+8,549021 x$ & 0,73 \\
\hline & Inflorescência & $y=10,594828+26,405097 x-67,089056 x^{2}$ & 0,99 \\
\hline \multirow{3}{*}{$\mathrm{Cu}$} & Raiz & $\begin{array}{l}y=1735,910383-191,031889 x- \\
3666,509813 x^{2}\end{array}$ & 0,89 \\
\hline & Parte aérea & $y=2071,860214-1262,509101 x$ & 0,77 \\
\hline & Inflorescência & $\begin{array}{l}y=385,334664- \\
1036,852693 x+1554,585512 x^{2}\end{array}$ & 0,86 \\
\hline \multirow{3}{*}{$\mathrm{Fe}$} & Raiz & s.a. & - \\
\hline & Parte aérea & $\begin{array}{l}y=160,682734- \\
301,765657 x+354,995432 x^{2}\end{array}$ & 0,87 \\
\hline & Inflorescência & $\begin{array}{l}y=79,806078- \\
945,814493 x+3230,949418 x^{2}\end{array}$ & 0,99 \\
\hline \multirow{3}{*}{$\mathrm{Mn}$} & Raiz & s.a. & - \\
\hline & Parte aérea & s.a. & - \\
\hline & Inflorescência & $y=55,061348+55,061348 x$ & 0,90 \\
\hline \multirow{3}{*}{$\mathrm{Zn}$} & Raiz & $y=59,929429-44,884332 x$ & 0,87 \\
\hline & Parte aérea & $y=36,414071-19,697120 x$ & 0,72 \\
\hline & Inflorescência & $y=39,010297+15,300867 x-45,51385 x^{2}$ & 0,99 \\
\hline \multirow{3}{*}{$\mathrm{Cr}$} & Raiz & $\begin{array}{l}y=279,230133+3128,997378 x- \\
2504,971674 x^{2}\end{array}$ & 0,80 \\
\hline & Parte aérea & $\begin{array}{l}y=10,153047- \\
268,448158 x+938,814947 x^{2}\end{array}$ & 0,99 \\
\hline & Inflorescência & $\begin{array}{l}y=49,881057- \\
1486,854811 x+5589,747406 x^{2}\end{array}$ & 0,99 \\
\hline
\end{tabular}

${ }^{* *}$ significativo a $5 \%$ de probabilidade; n.s. não-significativo a $5 \%$ de probabilidade; e s.a. sem ajuste. Fonte: próprios autores. 
$\mathrm{Na}$ raiz de arroz, os teores de $\mathrm{B}$ e Cu diminuíram até determinada dose de $\mathrm{Cr}$, passando a ser crescente a partir de então. Quanto ao teor de Zn, o mesmo diminuiu linearmente. $\mathrm{O}$ teor de $\mathrm{Cr}$ aumentou até a dose de 0,62 $\mathrm{mmol} \mathrm{L}^{-1}$ de $\mathrm{Cr}$, passando a diminuir depois dessa dose.

Ao analisar o teor de micronutrientes na parte aérea, constata-se que os teores de $\mathrm{Cu}$ e $\mathrm{Zn}$ sofreram diminuição com a presença de $\mathrm{Cr}$ em solução nutritiva, enquanto os teores de $\mathrm{Fe} \mathrm{e} \mathrm{Cr}$ apresentaram reposta quadrática, com os pontos mínimos alcançados nas doses de 0,06 e $0,14 \mathrm{mmol} \mathrm{L}^{-1}$ de $\mathrm{Cr}$.

Quanto aos teores de $\mathrm{Cu}, \mathrm{Fe}, \mathrm{Zn}$ e $\mathrm{Cr}$ da inflorescência, observa-se comportamento quadrático com o ponto máximo de $\mathrm{Cu}$ alcançado na dose de 0,33 mmol $\mathrm{L}^{-1}$ de $\mathrm{Cr}$, enquanto para $\mathrm{Fe}, \mathrm{Zn}$ e $\mathrm{Cr}$, os pontos mínimos foram alcançados nas doses de 0,$14 ; 0,17$ e $0,13 \mathrm{mmol} \mathrm{L}^{-1}$ de $\mathrm{Cr}$, respectivamente. $\mathrm{O}$ teor de $\mathrm{Mn}$ teve aumento linear em função do aumento da doses de $\mathrm{Cr}$ em solução.

Redondo-Gomez et al. (2011) estudando diferentes doses de Cr em Spartina argentinensis constataram que, com o aumento de $\mathrm{Cr}$ em solução, houve aumento no teor do mesmo. Os mesmos autores verificaram que, o teor de $\mathrm{P}$ e Cu diminuíam e o teor de B aumentava com o aumento da dose de $\mathrm{Cr}$ em solução nutritiva. Esses resultados corroboram aos observados no presente estudo.

Os acúmulos de macronutrientes na raiz, parte aérea e inflorescências foram influenciadas de forma significativa pela presença de $\mathrm{Cr}$ em solução nutritiva e são mostrados na Tabela 3. 
Tabela 3. Equações de regressão para os acúmulos de macronutrientes na raiz, na parte aérea e inflorescência de plantas de arroz, em resposta a doses crescentes de cromo (0 a $64 \mathrm{mmol}^{-1} \mathrm{Cr}$ ). UFLA, Lavras-MG.

\begin{tabular}{|c|c|c|c|}
\hline Nutriente & Parte da planta & Equação de regressão & $\mathrm{R}^{2}$ \\
\hline \multirow{3}{*}{ N } & Raiz & $\begin{array}{l}y=1013,288242+473,626832 x- \\
2761,146857 x^{2}\end{array}$ & 0,88 \\
\hline & Parte aérea & $\begin{array}{l}y=408,3854945-536,077376 x- \\
1735,668289 x^{2}\end{array}$ & 0,99 \\
\hline & Inflorescência & $y=507,7985-594,9625 x$ & 0,92 \\
\hline \multirow{3}{*}{$P$} & Raiz & $\begin{array}{l}y=195,518289+581,140771 x- \\
1359,378281 x^{2}\end{array}$ & 0,90 \\
\hline & Parte aérea & $y=59,080125+96,209997 x-274,750084 x^{2}$ & 0,89 \\
\hline & Inflorescência & $\begin{array}{l}y=79,300654- \\
328,073449 x+687,542649 x^{2}\end{array}$ & 0,94 \\
\hline \multirow{3}{*}{$K$} & Raiz & $y=781,997429-1157,021832 x$ & 0,86 \\
\hline & Parte aérea & $y=389,883-556,379435 x$ & 0,97 \\
\hline & Inflorescência & $y=146,370875-175,490625 x$ & 0,93 \\
\hline \multirow{3}{*}{$\mathrm{Ca}$} & Raiz & $y=267,806786-422,327189 x$ & 0,83 \\
\hline & Parte aérea & $y=94,337857-140,150922 x$ & 0,97 \\
\hline & Inflorescência & $y=20,217312-46,710937 x$ & 0,92 \\
\hline \multirow{3}{*}{$\mathrm{Mg}$} & Raiz & $y=111,132786-171,569931 x$ & 0,90 \\
\hline & Parte aérea & $y=85,592714-124,964747 x$ & 0,98 \\
\hline & Inflorescência & $y=41,732812-49,835937 x$ & 0,87 \\
\hline \multirow[b]{3}{*}{$S$} & Raiz & s.a. & - \\
\hline & Parte aérea & $y=41,332336+15,144427 x-124,973617 x^{2}$ & 0,97 \\
\hline & Inflorescência & $\begin{array}{l}y=38,781038- \\
114,143564 x+203,223868 x^{2}\end{array}$ & 0,94 \\
\hline
\end{tabular}

${ }^{* *}$ significativo a $5 \%$ de probabilidade; n.s. não-significativo a 5\% de probabilidade; e s.a. sem ajuste. Fonte: próprios autores.

Ao analisar o acúmulo radicular de $\mathrm{N}$ e $\mathrm{P}$, constata-se que houve resposta quadrática, com o mínimo nas doses de 0,09 e 0,21 mmol L-1 de Cr. Os acúmulos de $\mathrm{K}$, Ca e Mg diminuíram linearmente com o aumento da dose de $\mathrm{Cr}$ em solução de cultivo.

Quanto ao acúmulo de macronutrientes na parte aérea de arroz, houve resposta diferenciada. Assim, para os teores de $\mathrm{N}, \mathrm{P}$ e $\mathrm{S}$ houve diminuição até certa 
dose $\left(0,15 ; 0,18\right.$ e $0,46 \mathrm{mmol} \mathrm{L}^{-1}$ de $\mathrm{Cr}$, respectivamente), com aumento a partir de então. Para os teores de $\mathrm{K}, \mathrm{Ca}$ e $\mathrm{Mg}$ ocorreu resposta linear, diminuindo com o aumento da dose de $\mathrm{Cr}$ em solução nutritiva.

A aplicação de doses crescentes de $\mathrm{Cr}$ em solução nutritiva fez com que o acúmulo de N, K, Ca e Mg diminuíssem na inflorescência das plantas de arroz.

Os acúmulos de $\mathrm{Cr}$ e micronutrientes na raiz, parte aérea e inflorescências foram influenciados de forma significativa pela presença de $\mathrm{Cr}$ em solução nutritiva e são mostrados na tabela 4.

Tabela 4. Equações de regressão para os acúmulos de micronutrientes e de cromo na raiz, na parte aérea e inflorescência de plantas de arroz, em resposta a doses crescentes de cromo ( 0 a $64 \mathrm{mmol}^{-1} \mathrm{Cr}$ ). UFLA, Lavras-MG.

\begin{tabular}{|c|c|c|c|}
\hline Nutriente & Parte da planta & Equação de regressão & $\mathrm{R}^{2}$ \\
\hline \multirow{3}{*}{$\mathrm{B}$} & Raiz & $y=1049,823250+380,918918 x-3270,333501 x^{2}$ & 0,82 \\
\hline & Parte aérea & $y=475,258+103,871237 x-1192,019489 x^{2}$ & 0,97 \\
\hline & Inflorescência & $y=267,232562-302,067187 x$ & 0,90 \\
\hline \multirow{3}{*}{$\mathrm{Cu}$} & Raiz & $y=49659,215571-77657,668088 x$ & 0,85 \\
\hline & Parte aérea & $y=39565,199286-58779,369528 x$ & 0,97 \\
\hline & Inflorescência & $y=8478,908687-19591,339062 x$ & 0,92 \\
\hline \multirow{3}{*}{$\mathrm{Fe}$} & Raiz & $y=36472,745758-26791,64887 x-44101,16833 x^{2}$ & 0,90 \\
\hline & Parte aérea & $y=2956,353930-6139,418623 x+2879,495994 x^{2}$ & 0,95 \\
\hline & Inflorescência & n.s & - \\
\hline \multirow{3}{*}{$\mathrm{Mn}$} & Raiz & $y=1767,008141+4666,959423 x-11319,425456 x^{2}$ & 0,94 \\
\hline & Parte aérea & $y=2207,684516+2681,631618 x-8929,596407 x^{2}$ & 0,90 \\
\hline & Inflorescência & n.s & - \\
\hline \multirow{3}{*}{$\mathrm{Zn}$} & Raiz & $y=3700,434357-5659,067454 x$ & 0,97 \\
\hline & Parte aérea & $y=686,814214-973,072811 x$ & 0,98 \\
\hline & Inflorescência & $y=925,510250-1269,318750 x$ & 0,95 \\
\hline \multirow{3}{*}{$\mathrm{Cr}$} & Raiz & s.a. & - \\
\hline & Parte aérea & $y=20,739266+7,662565 x+885,259944 x^{2}$ & 0,99 \\
\hline & Inflorescência & $y=32,235885-418,784833 x+11863,135856 x^{2}$ & 0,99 \\
\hline
\end{tabular}

${ }^{* *}$ significativo a $5 \%$ de probabilidade; n.s. não-significativo a 5\% de probabilidade; e s.a. sem ajuste. Fonte: próprios autores. 
O aumento das doses de $\mathrm{Cr}$ influenciou os acúmulos de $\mathrm{B}, \mathrm{Cu}, \mathrm{Fe}, \mathrm{Mn}$ e $\mathrm{Zn}$ nas raízes. Resposta quadrática foi observada para $\mathrm{B}$, $\mathrm{Fe}$ e $\mathrm{Mn}$, com pontos máximos nas doses de 0,06 e 0,30 $\mathrm{mmol} \mathrm{L}^{-1}$ de $\mathrm{Cr}$ ( $\mathrm{B}$ e Fe, respectivamente) e ponto mínimo na dose de 0,21 $\mathrm{mmol} \mathrm{L}^{-1}$ de $\mathrm{Cr}$, para Mn. Para Cu e Zn, o acúmulo diminuiu com aumento da dose de $\mathrm{Cr}$.

Na parte aérea de plantas de arroz, houve resposta quadrática a aplicação de $\mathrm{Cr}$ no acúmulo de $\mathrm{B}, \mathrm{Fe}, \mathrm{Mn}$ e $\mathrm{C}$. Os acúmulos de $\mathrm{Cu}$ e $\mathrm{Zn}$ na parte aérea diminuíram linearmente em função do aumento das doses de $\mathrm{Cr}$.

Com aumento da dose de $\mathrm{Cr}$, houve queda nos acúmulos de $\mathrm{B}$, Cu e $\mathrm{Zn}$ nas inflorescências de arroz. Ainda para o acúmulo em inflorescência, o $\mathrm{Cr}$ apresentou o seu máximo na dose de $0,02 \mathrm{mmol} \mathrm{L}^{-1}$ de $\mathrm{Cr}$ e a partir desse ponto houve queda. A aplicação de $\mathrm{Cr}$ em solução nutritiva não exerceu efeito significativo para os acúmulos de $\mathrm{Fe}$ e $\mathrm{Mn}$ em infloresência. O acúmulo de $\mathrm{Cr}$ na raiz foi até 3000 vezes superior ao acumulado na parte área na dose de 0,04 $\mathrm{mmol} \mathrm{L}^{-1}$.

Através do índice de translocação (IT) é possível avaliar a capacidade das espécies em translocar os elementos ou nutrientes da raiz para a parte aérea. Observando o IT (Figura 4) verifica-se que, o $\mathrm{Cr}$ se transloca pouco do sistema radicular para a parte aérea, com índices que variam de 0,15 a $7,7 \%$, sendo este último para a dose $0,64 \mathrm{mmol} \mathrm{L}^{-1}$.

Figura 4: Índice de Translocação (IT) de Cr em plantas de arroz sob doses de cromo, Lavras-MG.

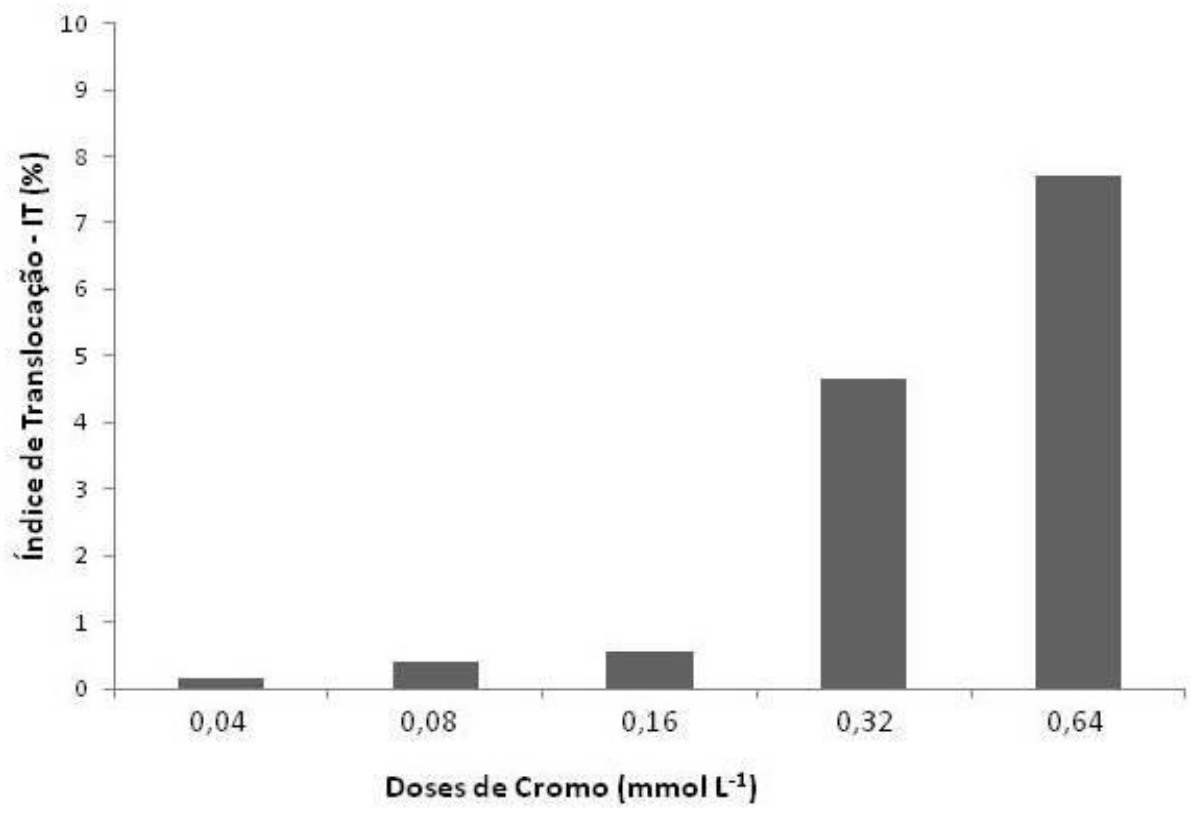

Fonte: próprios autores. 
As raízes acumularam a maior quantidade de $\mathrm{Cr}$ nas plantas de arroz, sendo responsável por mais de 92\% para todas as doses estudadas. Essa constatação se torna importante, pois havendo baixa translocação para os grãos haverá menor risco de toxicidade na cadeia trófica.

Estudos mostram que, o $\mathrm{Cr}$ é acumulado principalmente nas raízes, sendo uma pequena parte translocada para parte aérea. Isso se deve à imobilização desse elemento nos vacúolos das células radiculares, reduzindo seus efeitos tóxicos, o que pode ser uma resposta natural da planta à toxidez (SHANKER et al., 2005). Tal efeito é corroborado por Ferreira (2007) onde ele revela que o efeito tóxico do $\mathrm{Cr}$ no sistema vascular da raiz foi descrito em beterraba (Beta vulgaris L.) como responsável por danos no restante do organismo. Esta explicação baseia-se na sua baixa translocabilidade, o que leva a que a maior quantidade de $\mathrm{Cr}$ absorvido é acumulada nos tecidos da raiz. Relativamente à porção aérea, as concentrações mais baixas de $\mathrm{Cr}$ tendem a ser encontradas no fruto, medianamente no caule e mais elevadas concentrações nas folhas.

\section{Conclusões}

O aumento da concentração de $\mathrm{Cr}$ em solução nutritiva provoca diminuição na produção de matéria seca de arroz.

Os teores e acúmulos de nutrientes e $\mathrm{Cr}$ na raiz, parte aérea e inflorescência são influenciados pela presença de $\mathrm{Cr}$ em solução nutritiva, apresentando respostas diferenciadas.

O Cr é pouco translocado da raiz para a parte aérea em plantas de arroz.

\section{Referências}

BONFRANCESCHI, B. A.; FLOCCO, C. G.; DONATI, E. R. Study of the heavy metal phytoextraction capacity of two forage species growing in an hydroponic environment. New York, Journal of Hazardous Materials, v.165, p. 366-371, 2009.

CASTILHOS, D. D.; GUADAGNIN, C. A.; SILVA, M. Adição de cromo hexavalente no crescimento, nodulação e absorção de nutrientes em soja. Santa Maria, Ciência.

Rural, v.31, n.6, p.969-972, 2001 a. 
CASTILHOS, D. D.; GUADAGNIN, C. A.; SILVA, M. Acúmulo de cromo e seus efeitos na fixação biológica de nitrogênio e absorção de nutrientes em soja. Pelotas, Revista Brasileira de Agrociência, v.7, n.2, p.121-124, 2001 b.

CIPRIANI, H. N. Crescimento e acúmulo de cromo em mudas de Eucalyptus urograndis. Monografia, UFV- Lavras, 2008.

FERREIRA, D. F. SISVAR software: versão 5.6. Lavras: UFLA/DEX, 2010. Software

FERREIRA, A. L. N. Exposição de Crómio em alface: acumulação e efeitos na fisiologia e genotoxicidade. Universidade de Aveiro, Departamento de Biologia, Dissertação, Portugal 2007.

FERREIRA, E. V. O. et al. Manganês na nutrição mineral de cultivares de arroz de terras altas. Pesquisa Agropecuária Tropical v. 39, n. 2, p. 151-157, abr./jun. 2009.

FERREIRA, C. M.; VILLAR, P. M. D. Conjuntura socioeconômica da cultura do arroz em Minas Gerais e no Brasil. Informe Agropecuário, Belo Horizonte, v.39, n. 301, p.7-12, 2018.

LOPES, A. C. F. Formação de radicais livres induzida por cromo trivalente (Cr3+) e hexavalente (Cr6+). Dissertação, UNB Brasília, 2013.

GUPTA, V. K.; RASTOGI, A.; NAYAK, A. Adsorption studies on the removal of hexavalent chromium from aqueous solution using a low cost fertilizer industry waste material. Chicago, Journal of Colloid and Interface Science. v.342, p.135-141, 2010.

HOAGLAND, D.R.; ARNON, D.L. The water culture methods for growing plants without soil. Berkeley, California Agriculture Experiment Station. 1950. 32p. (Bulletin, 347).

KABATA-PENDIAS, A.; MUKHERJEE, A. B. Trace elements from soil to human. New York: Springer. 2007. 450p.

MALAVOLTA, E.; VITTI, G. C.; OLIVEIRA, S. A. Avaliação do estado nutricional das plantas: princípios e aplicações. Piracicaba: Potafos. 1997. 319p.

MARANGON, F. C.; FERNANDES, L. G. M. O uso do picolinato de cromo como coadjuvanteno tratamento da diabetes mellitus. Univ. Ci. Saúde, Brasília, v. 3, n. 2, p. 253-260, jul./dez. 2005.

MUNIZ, D. H. F.; OLIVEIRA-FILHO, E. C. Metais pesados provenientes de rejeitos de mineração e seus efeitos sobre a saúde e o meio ambiente. Universitas:

Ciências da Saúde. v. 4, n1/2, p.83-100, 2006.

PAIVA, H.N.; CARVALHO, J.G.; SIQUEIRA, J.O. Índice de translocação de nutrientes em mudas de cedro (Cedrela fissilis Vell.) e ipê-roxo (Tabeuia impetiginosa (Mart.) Standl.) submetidas a doses crescentes de cádmio, níquel e chumbo. Sociedade de Investigações Florestais, v. 26, p. 467-473, 2002. 
PANDA, S. K. E.; CHOUDHURY, S. Chromium stress in plants. California, Plant Physiology. v.17, n.1, p.95-102, 2005.

REDONDO-GOMEZ, S. et al. Accumulation and tolerance characteristics of chromium in a cordgrass Cr-hyperaccumulator, Spartina argentinensis. New York, Journal of Hazardous Materials, v.185, p.862-869, 2011.

SHANKER, A. K. et al. Chromium toxicity in plants. Lancaster, Environment International. v.31, p.739-753, 2005.

SOARES, C. R. F. S. et al. Acúmulo e distribuição de metais nas raízes, caule e folhas de mudas de árvores em solo contaminado por rejeitos de indústria de zinco. Campinas, Revista Brasileira de Fisiologia Vegetal, v.13, n.3, p.302-315, 2001. 